\title{
The relationship between MMP-2 and MMP-9 expression levels with breast cancer incidence and prognosis
}

\author{
HAI LI ${ }^{1 *}$, ZHENWEI QIU $^{1 *},{\text { FENG } \mathrm{LI}^{2} \text { and CHUNLEI WANG }}^{2}$ \\ ${ }^{1}$ Department of General Surgery, Yidu Central Hospital of Weifang, Weifang, Shandong 262500; \\ ${ }^{2}$ Department of General Surgery, Weifang People's Hospital, Weifang, Shandong 261041, P.R. China
}

Received May 29, 2017; Accepted September 5, 2017

DOI: $10.3892 / 01.2017 .6924$

\begin{abstract}
The relationship between the expression levels of matrix metalloproteinase-2 (MMP-2) and MMP-9 and breast cancer prognosis was studied. Two breast cancer cell lines (MDA-MB-231 and MCF-7) and one human normal breast cell line (HS578Bst) were investigated. Fluorescence real-time reverse transcription-polymerase chain reaction (RT-PCR) and western blotting were used to detect cellular mRNA and protein MMP-2 and MMP-9 expression levels. Breast cancer tissue samples from 80 patients and tumor-adjacent normal tissue samples from 40 patients were collected, and MMP-2 and MMP-9 expression in these samples were examined using immunohistochemistry (IHC). The relationship of MMP-2 and MMP-9 expression levels with breast cancer patient clinicopathological parameters and prognosis was analyzed. RT-PCR and western blot results showed that MMP-2 and MMP-9 mRNA and protein expression levels were significantly higher in MDA-MB-231 and MCF-7 cells than in HS578Bst cells. A high expression of MMP-2 and MMP-9 was found in 83.75\% $(67 / 80)$ and $78.75 \%(63 / 80)$ of breast cancer tissue samples, respectively. MMP-2 and MMP-9 expression in breast cancer tissues were significantly different from that in tumor-adjacent normal tissues ( $\mathrm{p}<0.01)$. MMP-2 and MMP-9 expression levels in breast cancer tissues were correlated with lymph node metastasis and tumor staging. Single factor survival analysis showed that MMP-2 and MMP-9 were factors influencing breast cancer prognosis. MMP-2 and MMP-9 are highly expressed in breast cancer tissues and are closely related to lymph node metastasis and tumor staging. MMP-2 and
\end{abstract}

Correspondence to: Dr Chunlei Wang, Department of General Surgery, Weifang People's Hospital, 151 Guangwen Street, Kuiwen, Weifang, Shandong 261041, P.R. China

E-mail: chunlei_wang1@163.com

*Contributed equally

Key words: matrix metalloproteinase-2, matrix metalloproteinase-9, breast cancer, prognosis
MMP-9 can be used as reference indices for guiding breast cancer prognosis and treatment.

\section{Introduction}

Breast cancer is a malignant tumor that often occurs in females, comprising $\sim 7-10 \%$ of all systemic malignant tumors. Tumor recurrence rate, metastasis rate, and mortality are high in patients with advanced breast cancer (1). As breast cancer is characterized by high rates of metastasis due to the presence of lymphatic vessels and reflux veins in the female breast, it is easy for breast cancer to transfer to the lung, liver, brain, and other organs during the early stages, resulting in poor clinical treatment and high mortality (2). In recent years, with developments in molecular biology and evidence-based medicine, treatment methods utilizing a combination of surgical resection with radiotherapy, chemotherapy, and targeted therapy has been developed to combat breast cancer. Although this comprehensive treatment method has a certain curative effect, the recurrence and metastasis rates for breast cancer patients remain high and prognosis is generally poor (3-5).

Matrix metalloproteinases (MMPs) are a family of zinc-dependent proteases, and MMP-2 and MMP-9 are major members of the MMP family (6,7). MMP-2 and MMP-9 are mainly secreted by tumor cells and stromal cells in the form of zymogens. After activation via hydrolysis, MMP-2 and MMP-9 degrade basement membrane (BM) type IV collagen, affecting the ability of BMs to impede tumor cell movement (8). Research has shown that MMP-2 and MMP-9 play key roles in degrading extracellular matrices and promoting tumor invasion and metastasis $(9,10)$.

The study aimed to investigate MMP-2 and MMP-9 expression levels in breast cancer and their relationships with breast cancer clinicopathological parameters and prognosis. In this study, two breast cancer cell lines (MDA-MB-231 and MCF-7) and one human normal breast cell line (HS578Bst) were cultured in vitro. Fluorescence RT-PCR and western blotting were used to detect the expressions of MMP-2 and MMP-9 in cells at mRNA and protein levels. MMP-2 and MMP-9 expression levels in breast cancer tissues and tumor-adjacent normal tissues were detected using immunohistochemistry (IHC) and the relationship between MMP-2 and MMP-9 expression and clinicopathological parameters and prognosis was studied. 


\section{Materials and methods}

Materials. Breast cancer cell lines MDA-MB-231 and MCF-7 and human normal breast cell line HS578Bst were acquired from the Cell Banks of the Chinese Academy of Sciences (Shanghai, China). The following reagents were used: Roswell Park Memorial Institute-1640 (RPMI-1640) medium and fetal bovine serum (FBS) (Gibco, Carlsbad, CA, USA); FBS (HyClone Laboratories, Logan, UT, USA); TRIzol kits, reverse transcription kits, and RT-PCR kits (Invitrogen, Carlsbad, CA, USA); MMP-2, MMP-9, glyceraldehyde 3-phosphate dehydrogenase (GAPDH) rabbit anti-human primary polyclonal antibodies (cat. no. 10373-2-AP, 10375-2-AP and 10494-1-AP), and mouse anti-rabbit horse-radish peroxidase (HRP) secondary monoclonal antibodies (cat. no. HRP-60004; Proteintech Group, Inc., Wuhan, China); bicinchoninic acid (BCA) protein quantification kits (Beyotime Institute of Biotechnology, Jiangsu, China); and IHC kit SP-9001 (Beijing Zhongshan Golden Bridge Biotechnology Co., Ltd., Beijing, China). Primer synthesis was performed by Takara (Dalian, China).

A total of 80 female breast cancer patients accompanied by complete clinical data admitted to Yidu Central Hospital of Weifang for treatment between January, 2007 and December, 2010 were selected for this study. Patients were aged 24-78 years (median age, 55 years). All patients were clinically and pathologically diagnosed with breast cancer and received surgical treatment for the first time without any prior history of receiving radiotherapy and chemotherapy. Informed consents were signed by the patients and/or guardians. The study was approved by the Ethics Committee of Yidu Central Hospital of Weifang. Breast cancer tissue samples were extracted from tumors by excision. Tumor-adjacent normal tissues were extracted from an area $10 \mathrm{~cm}$ away from the tumor edge (no infiltration of tumor cells was observed by microscopy). Specimens were fixed with $10 \%$ formaldehyde and embedded in conventional paraffin after extraction. All patients had complete follow-up records, including age, tumor size, lymph node status, clinical staging and survival condition.

Detection of cellular mRNA MMP-2 and MMP-9 expression by RT-PCR. HS578Bst, MCF-7 and MDA-MB-231 cells were cultured in RPMI-1640 medium containing 10\% FBS in an incubator at $37^{\circ} \mathrm{C}$ with $5 \% \mathrm{CO}_{2}$. The medium was changed every other day and cells were digested by Trypsin and then subcultured upon initiation of cell fusion.

Cells were collected during the logarithmic growth phase following digestion and centrifugation. Total RNA was extracted from the sample using TRIzol kits according to manufacturer's instructions. RNA concentration and purity were measured, with an A260/A280 value from 1.8-2.0 deemed acceptable. Reverse transcription was conducted according to the instructions provided in the reverse transcription kit, and mRNA expression was detected according to the methods provided in the RT-PCR kit using the RT-derived cDNA as the template (primer sequences are listed in Table I). GAPDH was selected as the internal reference. The reaction conditions were as follows: $94^{\circ} \mathrm{C}$ for $5 \mathrm{~min} ; 94^{\circ} \mathrm{C}$ for $30 \mathrm{sec}, 57^{\circ} \mathrm{C}$ for $30 \mathrm{sec}$, $72^{\circ} \mathrm{C}$ for $30 \mathrm{sec}$ for 30 cycles; $72^{\circ} \mathrm{C}$ for $5 \mathrm{~min}$. $\mathrm{Ct}$ values were generated by instrument software (ABI7300; Thermo Fisher
Table I. RT-PCR primer sequences.

\begin{tabular}{ll} 
Gene & \multicolumn{2}{c}{ Primer sequences } \\
\hline MMP-2 & F: 5'-CTCATCGCAGATGCCTGGAA-3' \\
& R: 5'-TTCAGGTAATAGGCACCCTTGAAGA-3' \\
MMP-9 & F: 5'-ACGCACGACGTCTTCCAGTA-3' \\
& R: 5'-CCACCTGGTTCAACTCACTCC-3' \\
GAPDH & F: 5'-GCACCGTCAAGGCTGAGAAC-3' \\
& R: 5'-TGGTGAAGACGCCAGTGGA-3' \\
\hline
\end{tabular}

MMP, matrix metalloproteinase; GAPDH, glyceraldehyde 3-phosphate dehydrogenase; F, forward; R, reverse.

Scientific, Waltham, MA, USA). Relative expression levels were calculated using the $2^{-\Delta \mathrm{Ct}}$ method according to the following formula: $\Delta \mathrm{Ct}$ (target gene) $=\mathrm{Ct}$ (target gene) $\mathrm{Ct}$ (control gene) .

Detection of cellular MMP-2 and MMP-9 expression by western blot analysis. Digestion and centrifugation were used to collect cells during the logarithmic growth phase. Cell lysates were generated and protein concentrations were determined by BCA assay. Protein samples $(50 \mu \mathrm{g})$ were then loaded and separated by sodium dodecyl sulfate polyacrylamide gel electrophoresis (SDS-PAGE). Isolated proteins were transferred to polyvinylidene difluoride (PVDF) membranes and blocked with 5\% skim milk powder at room temperature for $2 \mathrm{~h}$. Rabbit anti-human MMP-2, MMP-9, and GAPDH primary polyclonal antibodies $(1: 1,000)$ were added, respectively, and membranes were incubated overnight at $4^{\circ} \mathrm{C}$. Membranes were then completely washed by Tris-buffered saline with Tween-20 (TTBS) and mouse anti-rabbit secondary monoclonal antibodies $(1: 2,000)$ were added, with the membrane incubated for $1 \mathrm{~h}$ at room temperature. Enhanced chemiluminescence (ECL) development was conducted in the dark, with a gel imager (Bio-Rad Laboratories, Hercules, CA, USA) used to obtain results. GADPH was used as an internal reference and gray scale analysis was performed.

Detection of MMP-2 and MMP-9 protein expression in pathological tissues by IHC. The procedure was carried out according to the instructions of the IHC kit. Briefly, after paraffin sections were dewaxed, endogenous peroxidases were inactivated with $3 \% \mathrm{H}_{2} \mathrm{O}_{2}$. Citrate buffer was used for thermal remediation and proteins were blocked with $10 \%$ goat serum. MMP-2 and MMP-9 primary antibodies (1:100 dilution) were applied, respectively, and samples were incubated at $4^{\circ} \mathrm{C}$ overnight. After washing with phosphate-buffered saline (PBS) three times, biotin-labeled secondary antibodies were added, with samples allowed to incubate for $15 \mathrm{~min}$. After that, proteins were washed with PBS three times and diaminobenzidine (DAB) solution was applied for color development in the dark. Hematoxylin was used for restaining, and gum was used for mounting. Finally, pictures were taken under a microscope (TE2000-U; Nikon, Tokyo, Japan).

MMP-2 and MMP-9 protein expression was noted in the cytoplasm by the presence of a brown color. The proportion 
Table II. MMP-2 and MMP-9 protein expression levels in breast cancer and tumor-adjacent normal tissues (cases, \%).

\begin{tabular}{lcrrrrr}
\hline & & \multicolumn{2}{c}{ MMP-2 } & & \multicolumn{2}{c}{ MMP-9 } \\
\cline { 3 - 4 } Group & Cases & - and + & ++ and +++ & & - and + & ++ and +++ \\
\hline Breast cancer tissues & 80 & $13(16.25)$ & $67(83.75)$ & & $17(21.25)$ & $63(78.75)$ \\
Tumor-adjacent normal tissues & 40 & $31(77.50)$ & $9(22.50)$ & & $33(82.50)$ & $7(17.50)$ \\
$\chi^{2}$-value & & & 43.08 & & 41.16 \\
P-value & & $<0.01$ & & $<0.01$ \\
\hline
\end{tabular}

MMP, matrix metalloproteinase.

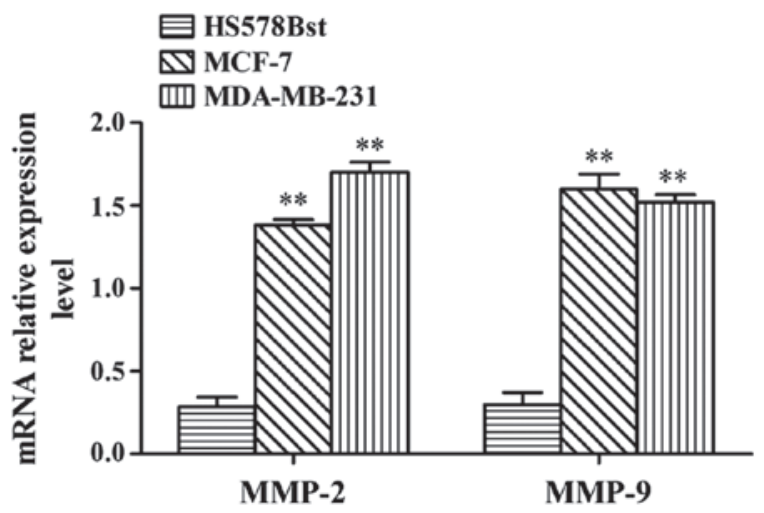

Figure 1. Detection of matrix metalloproteinase-2 (MMP-2) and MMP-9 mRNA expression in MCF-7, MDA-MB-231 and HS578Bst cells. The expression levels of MMP-2 and MMP-9 mRNA in MDA-MB-231 and MCF-7 cells were significantly higher than in HS578Bst cells, ${ }^{* *} \mathrm{p}<0.01$.

of positive cells among total cells in the entire visual field were recorded and divided into four categories: $<10 \%(-)$; $10-25 \%(+) ; 26-75 \%(++) ;>75 \%(+++)$. Categories - and + represent low expression while ++ and +++ represent high expression. Scores were calculated and analyzed.

Examining the relationship between MMP-2 and MMP-9 expression and breast cancer prognosis. Patients were divided into MMP-2 low expression and high expression groups as well as MMP-9 low and high expression groups according to the IHC results. There were no statistically significant differences in terms of gender, smoking history, alcohol history, and family history among these groups. Regular follow-ups were conducted after surgery for 5 years in the form of phone calls or outpatient reviews. Survival duration was recorded from the first day after surgery to the patient death or the follow-up conclusion deadline. MMP-2 and MMP-9 expression effects on breast cancer patient survival conditions were analyzed statistically based on follow-up results.

Statistical analysis. Statistical Product and Service Solutions (SPSS) 17.0 (International Business Machines Corp., Armonk, NY, USA) was used in this study. Measurement data were analyzed by using one-way analysis of variance (ANOVA). The data were compared between the two groups using $\chi^{2}$ analysis. Clinical prognostic data were analyzed by Kaplan-Meier survival analysis. $\mathrm{p} \leq 0.05$ was interpreted as a statistically significant difference.

\section{Results}

MMP-2 and MMP-9 mRNA expression levels in MCF-7, MDA-MB-231 and HS578Bst cells. RT-PCR results showed that MMP-2 and MMP-9 mRNA expression levels in MDA-MB-231 and MCF-7 cells were significantly higher than in HS578Bst cells ( $\mathrm{p}<0.01)$ (Fig. 1).

MMP-2 and MMP-9 protein expression in MCF-7, MDA-MB-231 and HS578Bst cells. Western blot results showed that MMP-2 and MMP-9 protein expression in MDA-MB-231 and MCF-7 cells were significantly higher than those in HS578Bst cells (p<0.01) (Fig. 2). Representative western blot analyses for MMP-2 and MMP-9 protein expression (Fig. 2A). Gray-scale densitometry analysis results for MMP-2 and MMP-9 protein expression (Fig. 2B). MMP-2 and MMP-9 protein expression in MDA-MB-231 and MCF-7 cells were significantly higher than in HS578Bst cells, $\mathrm{p}<0.01$.

MMP-2 and MMP-9 protein expression in breast cancer tissues. IHC results showed that the positive expression of MMP-2 and MMP-9 appeared as brown particles in the cytoplasm (Fig. 3). Through statistical analysis, it was found that high expression rates of MMP-2 and MMP-9 were found in the tumors of $83.75 \%(67 / 80)$ and $78.75 \%(63 / 80)$ of the patients enrolled in this study, respectively. Differences in MMP expression between breast cancer tissues and tumor-adjacent normal tissues were statistically significant $(\mathrm{p}<0.01)$ (Table II).

The relationship between breast cancer clinicopathological indices and MMP-2 and MMP-9 expression levels. Table III shows the analysis results of the relationship between breast cancer clinicopathological indices and MMP-2 and MMP-9 expression. Abnormal expression levels of MMP-2 and MMP-9 were correlated with the occurrence of lymph node metastasis and tumor staging $(\mathrm{p}<0.05)$, but not correlated with patient age and tumor size $(\mathrm{p}>0.05)$.

Analysis of survival condition and breast cancer patient prognosis. A total of 80 patients with breast cancer were followed up, in which 51 patients survived and 29 patients died (Table IV). Kaplan-Meier single factor analysis showed that MMP-2 and MMP-9 expression were associated with a significant effect on patient prognosis $(\mathrm{p}<0.05)$. Low MMP-2 and MMP-9 expression indicated a relatively good patient prognosis (Table IV and Fig. 4). 
Table III. The relationship between abnormal MMP-2 and MMP-9 expression and clinicopathological parameters.

\begin{tabular}{|c|c|c|c|c|c|c|c|}
\hline \multirow[b]{2}{*}{ Clinical parameter } & \multirow[b]{2}{*}{ Cases } & \multicolumn{3}{|c|}{ MMP-2 positive } & \multicolumn{3}{|c|}{ MMP-9 positive } \\
\hline & & Cases, $\%$ & $\chi^{2}$-value & P-value & Cases, $\%$ & $\chi^{2}$-value & P-value \\
\hline \multicolumn{8}{|l|}{ Age (years) } \\
\hline$\leq 40$ & 33 & $28(84.85)$ & 0.05 & $>0.05$ & $25(75.76)$ & 0.30 & $>0.05$ \\
\hline$>40$ & 47 & $39(82.98)$ & & & $38(80.85)$ & & \\
\hline \multicolumn{8}{|l|}{ Tumor size $(\mathrm{cm})$} \\
\hline$\leq 5$ & 49 & $42(85.71)$ & 0.36 & $>0.05$ & $38(77.55)$ & 0.11 & $>0.05$ \\
\hline$>5$ & 31 & $25(80.65)$ & & & $25(80.65)$ & & \\
\hline \multicolumn{8}{|c|}{ Lymphatic metastasis } \\
\hline No & 34 & $25(73.53)$ & 4.45 & $<0.05$ & $23(67.65)$ & 4.36 & $<0.05$ \\
\hline Yes & 46 & $42(91.30)$ & & & $40(86.96)$ & & \\
\hline \multicolumn{8}{|l|}{ Tumor staging } \\
\hline I-II & 29 & $20(68.97)$ & 5.70 & $<0.05$ & $18(62.07)$ & 7.56 & $<0.05$ \\
\hline III-IV & 51 & $47(92.16)$ & & & 45 (88.24) & & \\
\hline
\end{tabular}

MMP, matrix metalloproteinase.

Table IV. Relationship between patient survival condition and MMP-2 and MMP-9 expression.

\begin{tabular}{lccccc}
\hline Group & Cases & 5-year-survival (cases) & 5-year-survival (\%) & Wald (log-rank) & P-value \\
\hline MMP-2 & & 39 & 58.21 & 5.007 & $<0.05$ \\
High expression & 67 & 12 & 92.31 & & \\
Low expression & 13 & & & 5.584 & $<0.05$ \\
MMP-9 & 63 & 36 & 57.14 & & \\
High expression & 15 & 15 & 88.24 & & \\
Low expression & 17 & &
\end{tabular}

MMP, matrix metalloproteinase.

A

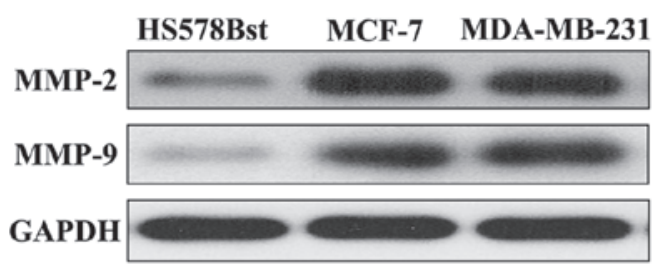

B

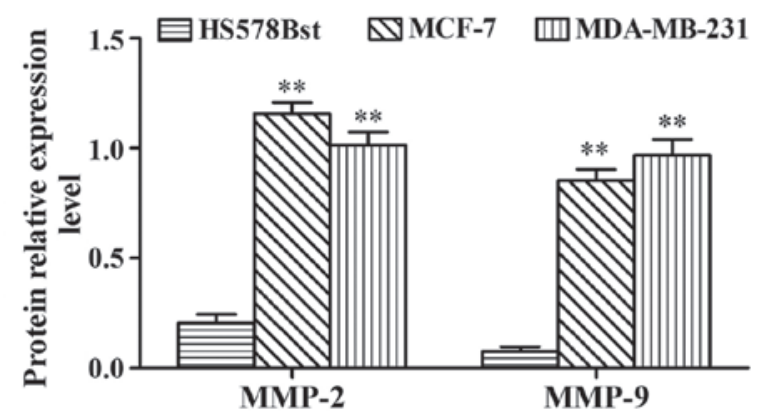

Figure 2. (A and B) Detection of matrix metalloproteinase-2 (MMP-2) and MMP-9 protein expression in MCF-7, MDA-MB-231 and HS578Bst cells by western blot analysis $(* * \mathrm{p}<0.05)$.

\section{Discussion}

Breast cancer is a malignant tumor occurring in breast epithelial tissues, and its pathogenesis is very complex. In recent years, the incidence rate of breast cancer has increased on an annual basis, and more young people have developed the disease. Breast cancer has become an important factor affecting women's health (11-13).

The MMP-2 gene is located on the human chromosome 16q21. Activated MMP-2 can not only degrade type IV collagen in BMs, but also can degrade type V, VI and X collagens as well as gelatins (14). MMP-9 can be secreted 


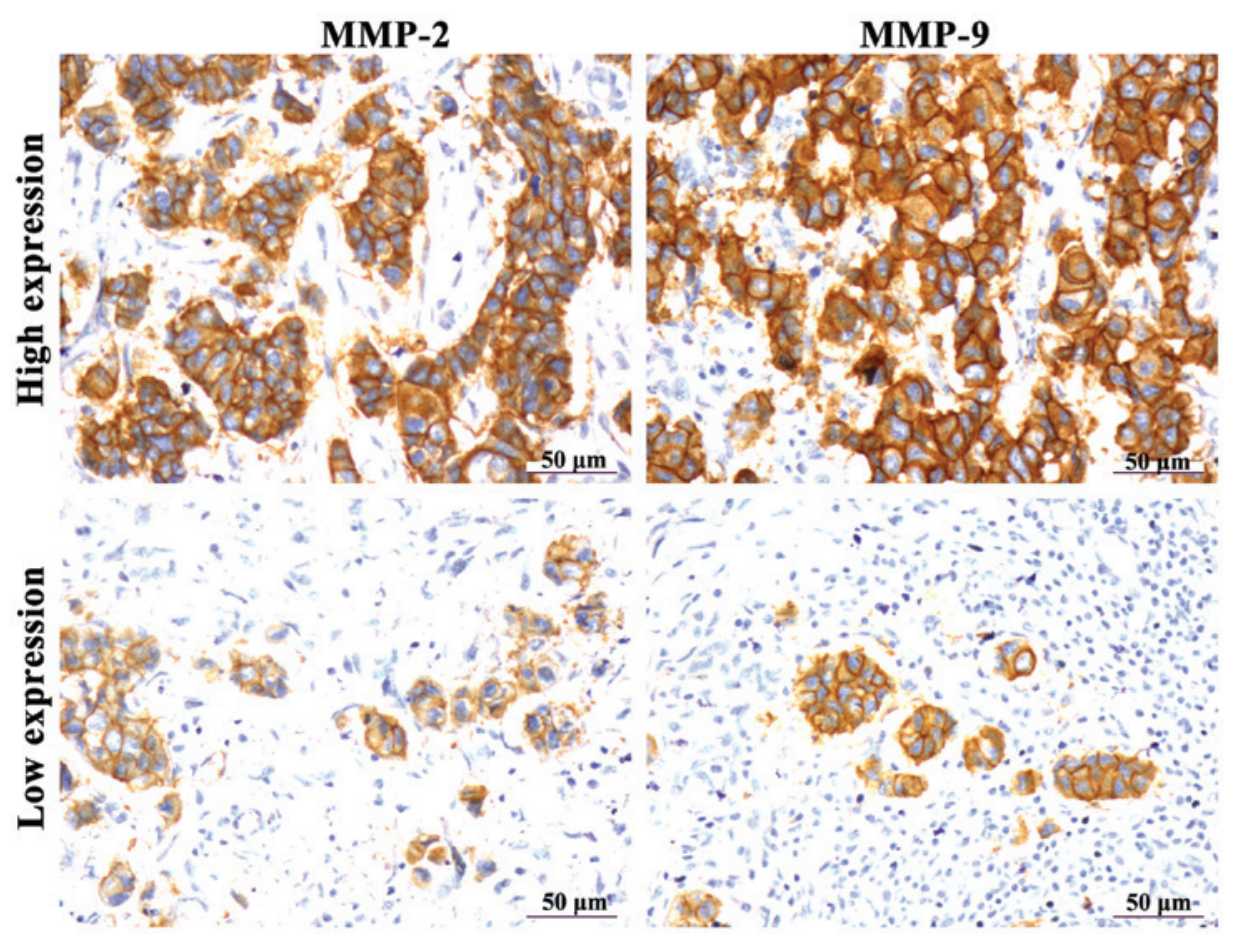

Figure 3. Detection of matrix metalloproteinase-2 (MMP-2) and MMP-9 protein expression in clinicopathological tissues via immunohistochemistry (IHC) MMP-2 and MMP-9 are expressed in the cytoplasm and appear as brown granules; a proportion of positive cells $>25 \%$ in the visual field represented high expression, otherwise, the result was categorized as low expression ( $\mathrm{x} 400)$.
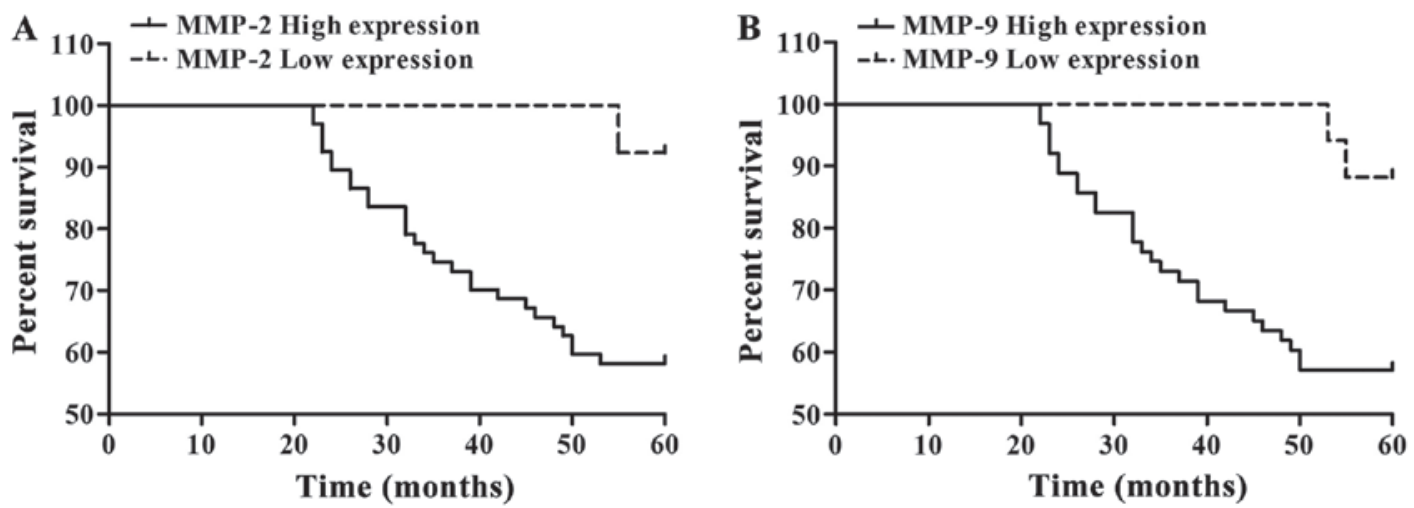

Figure 4. (A and B) Survival curves for patients with high and low expression levels of matrix metalloproteinase-2 (MMP-2) and MMP-9 Kaplan-Meier analysis shows that patients with low expression of MMP-2 and MMP-9 showed improved prognoses.

extracellularly in the form of zymogen, and activated MMP-9 can degrade type IV collagens and fibronectins to destroy BMs and extracellular matrixes, thereby affecting the adhesion ability of tumor cells (15). Extracellular matrixes and BMs are natural barriers in tumor infiltration and diffusion processes, and cancer cells passing through these physical barriers is key to tumor cell transfer and migration. It has been found that type IV collagens are the main components of the extracellular matrix and BMs. Tumor cells are able to specifically express MMP-2 and MMP-9 to degrade type IV collagens and destroy these tissue barriers, thus promoting the invasion and metastasis of tumor cells (16). According to studies, MMP-2 and MMP-9 expression levels in breast cancer tissues were significantly higher than in fibrous adenomas, and likewise, tumor tissue MMP-2 and MMP-9 expression was significantly higher in patients with infiltrative breast cancer and lymph node metastasis than patients with non-infiltrative cancer and non-lymph node metastasis $(17,18)$. Based on 168 cases of postmenopausal advanced breast cancer, Rahko et al found that MMP-9 expression was closely related to clinical staging, pathology type, and hormone receptor status (19). Li et al (20) used IHC to detect MMP-2 and MMP-9 expression levels in 270 cases of axillary lymph node-negative breast cancer, finding that MMP-2 and MMP-9 expression were positively correlated with histological grading.

This study investigated the effects of MMP-2 and MMP-9 on breast cancer patient pathological parameters and prognosis. First, the breast cancer cell lines MDA-MB-231 and MCF-7, as well as the human normal breast cell line HS578Bst were selected and cultured. MMP-2 and MMP-9 mRNA and protein expression levels were significantly higher in the breast cancer cell lines than in HS578Bst cells. Following this, breast 
cancer tissue specimens were collected from 80 patients, with tumor-adjacent normal tissue specimens collected from 40 patients. MMP-2 and MMP-9 expression in breast cancer tissues were significantly higher than in tumor-adjacent normal tissues. High expression levels of MMP-2 and MMP-9 were correlated with lymph node metastasis and tumor staging by clinicopathological data analysis. Kaplan-Meier single factor survival analysis showed that low MMP-2 and MMP-9 expression indicated relatively good patient prognosis.

In recent years, the relationship between MMPs and breast cancer has become a research hotspot. Jones et al found that MMP-2 was mainly expressed in the cytoplasm of breast cancer cells, and a small amount of these cells were present in the normal breast duct and the BMs around acini (21). Lebeau et al detected MMP-2 and MMP-9 expression in 70 infiltrative breast cancer patients, finding that MMP-2 and MMP-9 were expressed in tumor cells and surrounding stromal cells, with particularly high expression at the edges of the tumor infiltration area (22). This study not only confirmed the presence of high MMP-2 and MMP-9 expression in breast cancer cell lines and breast cancer tissues, but also confirmed that MMP-2 and MMP-9 expression were associated with lymph node metastasis, tumor staging and prognosis.

In conclusion, MMP-2 and MMP-9 are highly expressed in breast cancer and are closely related to lymph node metastasis and tumor staging. MMP-2 and MMP-9 can be used as reference indicators for guiding breast cancer treatment and estimating prognosis.

\section{References}

1. Jemal A, Bray F, Center MM, Ferlay J, Ward E and Forman D: Global cancer statistics. CA Cancer J Clin 61: 69-90, 2011.

2. Garcia-Murillas I, Schiavon G, Weigelt B, Ng C, Hrebien S, Cutts RJ, Cheang M, Osin P, Nerurkar A, Kozarewa I, et al: Mutation tracking in circulating tumor DNA predicts relapse in early breast cancer. Sci Transl Med 7: 302ra133, 2015.

3. Im NK, Jang WJ, Jeong CH and Jeong GS: Delphinidin suppresses PMA-induced MMP-9 expression by blocking the NF- $\kappa$ B activation through MAPK signaling pathways in MCF-7 human breast carcinoma cells. J Med Food 17: 855-861, 2014.

4. Shalaby MA, Nounou HA, Ms A, O A, Azzam N and Saeed HM: Associations between single nucleotide polymorphisms of COX-2 and MMP-2 genes and colorectal cancer susceptibility in the Saudi population. Asian Pac J Cancer Prev 15: 4989-4994, 2014.

5. Zhou R, Xu L, Ye M, Liao M, Du H and Chen H: Formononetin inhibits migration and invasion of MDA-MB-231 and 4T1 breast cancer cells by suppressing MMP-2 and MMP-9 through PI3K AKT signaling pathways. Horm Metab Res 46: 753-760, 2014.

6. Chia CY, Kumari U and Casey PJ: Breast cancer cell invasion mediated by Ga12 signaling involves expression of interleukins- 6 and -8, and matrix metalloproteinase-2. J Mol Signal 9: 6, 2014.

7. Stoeltzing O, Ahmad SA, Liu W, McCarty MF, Wey JS, Parikh AA, Fan F, Reinmuth N, Kawaguchi M, Bucana CD, et al: Angiopoietin-1 inhibits vascular permeability, angiogenesis, and growth of hepatic colon cancer tumors. Cancer Res 63: 3370-3377, 2003.
8. Zhang W, Wang F, Xu P, Miao C, Zeng X, Cui X, Lu C, Xie H, Yin $\mathrm{H}$, Chen $\mathrm{F}$, et al: Perfluorooctanoic acid stimulates breast cancer cells invasion and up-regulates matrix metalloproteinase-2/-9 expression mediated by activating NF- $\kappa$ B. Toxicol Lett 229: 118-125, 2014.

9. Iochmann S, Bléchet C, Chabot V, Saulnier A, Amini A, Gaud G, Gruel Y and Reverdiau P: Transient RNA silencing of tissue factor pathway inhibitor-2 modulates lung cancer cell invasion. Clin Exp Metastasis 26: 457-467, 2009.

10. Safranek J, Pesta M, Holubec L, Kulda V, Dreslerova J, Vrzalova J, Topolcan O, Pesek M, Finek J and Treska V: Expression of MMP-7, MMP-9, TIMP-1 and TIMP-2 mRNA in lung tissue of patients with non-small cell lung cancer (NSCLC) and benign pulmonary disease. Anticancer Res 29: 2513-2517, 2009.

11. Huang S, Chen J and Jia Y: Research on the correlation of MMP9 and $\mathrm{p} 53$ expression with the prognosis of triple negative breast cancer. Xiandai Shengwu Yixue Jinzhan 14: 881-884, 2014.

12. Choi JS, Baek HM, Kim S, Kim MJ, Youk JH, Moon HJ, Kim EK and Nam YK: Magnetic resonance metabolic profiling of breast cancer tissue obtained with core needle biopsy for predicting pathologic response to neoadjuvant chemotherapy. PLoS One 8: e83866, 2013

13. Yari K, Rahimi Z, Moradi MT and Rahimi Z: The MMP-2 -735 $\mathrm{C}$ allele is a risk factor for susceptibility to breast cancer. Asian Pac J Cancer Prev 15: 6199-6203, 2014.

14. Fernandez-Garcia B, Eiró N, Marín L, González-Reyes S, González LO, Lamelas ML and Vizoso FJ: Expression and prognostic significance of fibronectin and matrix metalloproteases in breast cancer metastasis. Histopathology 64: 512-522, 2014.

15. Li H, Huang J, Yang B, Xiang T, Yin X, Peng W, Cheng W, Wan J, Luo F, Li H, et al: Mangiferin exerts antitumor activity in breast cancer cells by regulating matrix metalloproteinases, epithelial to mesenchymal transition, and $\beta$-catenin signaling pathway. Toxicol Appl Pharmacol 272: 180-190, 2013.

16. Parsons SL, Watson SA, Collins HM, Griffin NR, Clarke PA and Steele RJ: Gelatinase (MMP-2 and -9) expression in gastrointestinal malignancy. Br J Cancer 78: 1495-1502, 1998.

17. Bucan V, Mandel K, Bertram C, Lazaridis A, Reimers K, Park-Simon TW, Vogt PM and Hass R: LEF-1 regulates proliferation and MMP-7 transcription in breast cancer cells. Genes Cells 17: 559-567, 2012.

18. Katunina AI, Gershtein ES, Ermilova VD, Tereshkina IV, Nazarenko AY, Tyleuova AA, Dvorova EK, Karabekova ZK, Gritskevich MV and Berezov TT: Matrix metalloproteinases 2, 7 , and 9 in tumors and sera of patients with breast cancer. Bull Exp Biol Med 151: 359-362, 2011.

19. Rahko E, Jukkola A, Melkko J, Paavo P, Bloigu R, Talvensaari-Mattila A and Turpeenniemi-Hujanen T: Matrix metalloproteinase-9 (MMP-9) immunoreactive protein has modest prognostic value in locally advanced breast carcinoma patients treated with an adjuvant antiestrogen therapy. Anticancer Res 24: 4247-4253, 2004.

20. Li HC, Cao DC, Liu Y, Hou YF, Wu J,Lu JS, Di GH,Liu G, Li FM, Ou ZL, et al: Prognostic value of matrix metalloproteinases (MMP-2 and MMP-9) in patients with lymph node-negative breast carcinoma. Breast Cancer Res Treat 88: 75-85, 2004.

21. Jones JL, Glynn P and Walker RA: Expression of MMP-2 and MMP-9, their inhibitors, and the activator MT1-MMP in primary breast carcinomas. J Pathol 189: 161-168, 1999.

22. Lebeau A, Müller-Aufdemkamp C, Allmacher C, Sauer U, Nerlich A, Lichtinghagen R and Löhrs U: Cellular protein and mRNA expression patterns of matrix metalloproteinases-2, -3 and -9 in human breast cancer: Correlation with tumour growth J Mol Histol 35: 443-455, 2004. 